
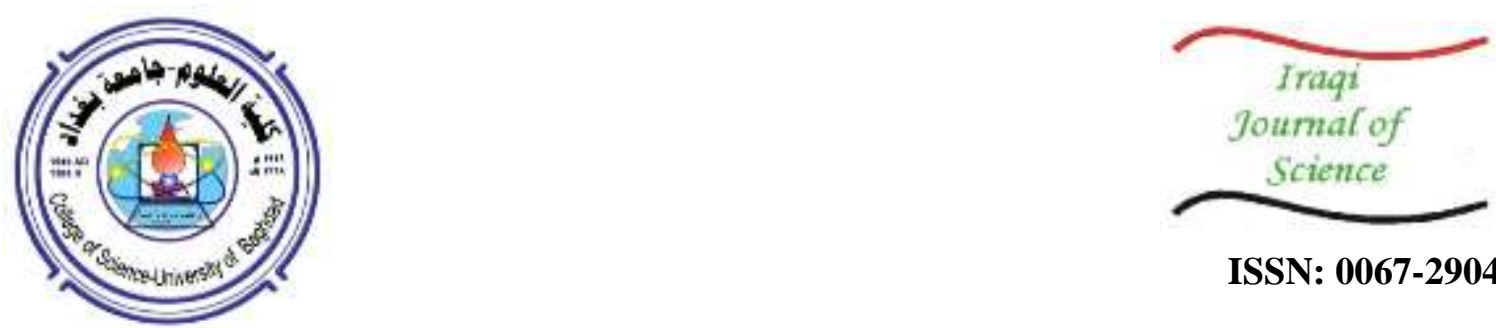

ISSN: 0067-2904

\title{
A certain Subclass of Meromorphically Multivalent Q-Starlike Functions Involving Higher-Order Q-Derivatives
}

\author{
Mustafa I. Hameed ${ }^{{ }^{*} \text {, Muna H. Ali }}{ }^{1}$, Buthyna Najad Shihab ${ }^{2}$ \\ ${ }^{l}$ Department of Mathematics, College of Education for Pure Sciences, University of Anbar, Anbar, Iraq \\ ${ }^{2}$ University of Baghdad, College of Education for Pure Sciences, Ibn Al-Haitham, Department of Mathematics, \\ Baghdad, Iraq \\ Received: 17/4/2021 Accepted: $11 / 7 / 2021$

\begin{abstract}
The authors introduced and addressed several new subclasses of the family of meromorphically multivalent $q$-star-like functions in the punctured unit disk $\mathcal{L}^{*}$ in this study, which makes use of several higher order $q$-derivatives. Many fascinating properties and characteristics are extracted systematically for each of these newly identified function classes. Distortion theorems and radius problems are among these characteristics and functions. A number of coefficient inequalities for functions belonging to the subclasses are studied, and discussed, as well as a suitable condition for them is set. The numerous results are presented in this study and the previous works on this subject are also connected together in this study.
\end{abstract}

Keywords: Multivalent functions, Meromorphically starlike functions, $q$ derivative(or $q$-difference) operator, Distortion theorems, Radius of $q$-starlikeness, close-to-convexity, convexity, Hadamard product.

\section{صنف جزئي معين من الدوال النجمية المتعددة التكافؤ الميرومورفية التي تتضمن مشتقات عليا من

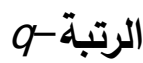

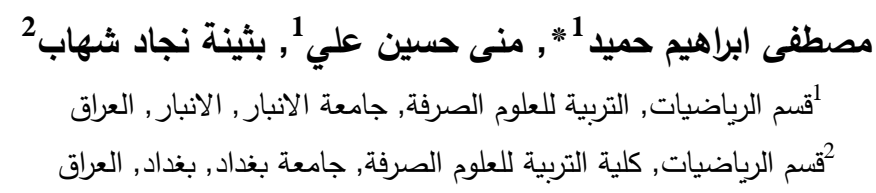




\section{Introduction and Definitions}

Let $\mathcal{L}=\{w \in \mathbb{C}:|w|<1\}$, and $H(\mathcal{L})$ are an open unit disc in $\mathbb{C}$, and the analytic functions class in $\mathcal{L}$, respectively. Suppose that $\mathcal{L}[a, i]$ is the subclass of $H(\mathcal{L})$ of the form

$$
g(w)=a+a_{\iota} w^{\iota}+a_{\iota+1} w^{\iota+1}+\cdots,
$$

where $a \in \mathbb{C}$ and $\iota \in \mathbb{N}=\{1,2, \ldots\}$ with $H_{0} \equiv H[0,1]$ and $H \equiv H[1,1]$. Let $g(w)$ be an analytic function in the punctured unit disk $\mathcal{L}^{*}$. If the equation $v=g(w)$ has never more than $u$-solutions in $\mathcal{L}^{*}=\{w: w \in \mathbb{C}$ and $0<|w|<1\}=\mathcal{L} \backslash\{0\}$, then $g(w)$ is said to be meromorphic $u$-valent in $\mathcal{L}^{*}$.

$M \Sigma(u)$ denotes the class of all analytical meromorphic $u$-valent functions where $g$ is represented by the forms.

$$
g(w)=w^{-u}+\sum_{\iota=1}^{\infty} a_{\iota} w^{\iota-u}, \quad\left(u, \iota \in \mathbb{N}=\{1,2,3, \ldots\}, w \in \mathcal{L}^{*}\right) .
$$

The subclasses $\Sigma^{*}(u)$ of the class $M \Sigma(u)$ are meromorphically multivalent starlike functions. A function $g \in \Sigma^{*}(u)$ of the from (1.1) if

$$
\mathfrak{R} e\left\{-\frac{w g^{\prime}(w)}{g(w)}\right\}>0, \quad w \in \mathcal{L}^{*}
$$

Various papers have been devoted to the study of analytical function subfamilies, especially many of them are meromorphically multivalent function subfamilies. Meromorphic multivalent function boundary coefficients have recently been considered in [1]. For more details we refer to $[2,3,4]$, while neighborhoods of such meromorphic multivalent functions with negative coefficients have been studied in $[5,6,7,8,9]$. We refer the reader to [10-13] for certain convolution (or Hadamard product) properties for the meromorphic $p$-valent functions' convexity and starlikeness.

Some main principle information and the $q$-difference calculus are elicited in order to provide a better interpretation of the current paper. In this article we assume that unless otherwise specified,

$$
(0<q<1, \quad u \in \mathbb{N}=\{1,2,3, \ldots\}) \text {. }
$$

Let a subset of $\Sigma^{*}(u)$, which consists of negative coefficient functions, be $\mathcal{M}(u)$ and have the following representation of the series:

$$
g(w)=w^{-u}-\sum_{\iota=1}^{\infty}\left|a_{\iota}\right| w^{\iota-u}, \quad\left(u, \iota \in \mathbb{N}=\{1,2,3, \ldots\}, w \in \mathcal{L}^{*}\right) .
$$

Definition (1.1):[14,15] Let $q \in(0,1)$, the $q$-number $[\eta]_{q}$ is defined by the following

$$
[\eta]_{q}=\left\{\begin{array}{lc}
\frac{1-q^{\eta}}{1-q} & (\eta \in \mathbb{C}) \\
\sum_{k=0}^{-1} q^{n}=1+q+q^{2}+\cdots+q^{\iota-1} & (\eta=\iota \in \mathbb{N})
\end{array}\right\}
$$

Definition (1.2): $[16,17]$ In a given subset of $\mathbb{C}$, the $q$-derivative (or $q$-difference) $\mathrm{T}_{q}$ of a function is defined by

if $g^{\prime}(0)$ exists.

$$
\left(\mathrm{T}_{q} g\right)(w)=\left\{\begin{array}{lc}
\frac{g(w)-g(q w)}{(1-q) w} & (w \neq 0) \\
g^{\prime}(0) & (w=0)
\end{array}\right\},
$$


From equation (1.3), it is readily observed that

$$
\lim _{q \rightarrow 1-}\left(\mathrm{T}_{q} g\right)=\lim _{q \rightarrow 1-} \frac{g(w)-g(q w)}{(1-q) w}=g^{\prime}(w),
$$

In a particular subset of the set $\mathbb{C}$, for a differentiable function $g$. Furthermore, on the basis of equation (1.1) and equation(1.3), we obtain

$$
\begin{gathered}
\left(\mathrm{T}_{q}^{(1)} g\right)(w)=[-u]_{q} w^{-u-1}+\sum_{\iota=1}^{\infty}[\iota-u]_{q} a_{\iota} w^{\iota-u-1} \\
\left(\mathrm{~T}_{q}^{(2)} g\right)(w)=[-u]_{q}[-u-1]_{q} w^{-u-2}+\sum_{\iota=1}^{\infty}[\iota-u]_{q}[\iota-u-1]_{q} a_{\iota} w^{\iota-u-2} \\
\cdot \\
\cdot \\
\cdot\left(\mathrm{T}_{q}^{(-u)} g\right)(w)=[-u]_{q} !+\sum_{\iota=1}^{\infty} \frac{[\iota-u]_{q} !}{[\iota]_{q} !} a_{\iota} w^{\iota},
\end{gathered}
$$

where $\left(\mathrm{T}_{q}^{(-u)} g\right)(w)$ is the q-derivative of $g(w)$ of order $u$. For any non-negative integer $\iota$.

Now, for each $g \in \Sigma^{*}(u)$, when $i$ times are applied to both sides of (1.1) with respect to $w$, it is easily seen by the operator $\mathrm{T}_{q}$ that

$$
\left(\mathrm{T}_{q}^{(i)} g\right)(w)=\frac{[-u]_{q} !}{[-u-i]_{q} !} w^{-u-i}+\sum_{\iota=1}^{\infty} \frac{[\iota-u]_{q} !}{[\iota-u-i]_{q} !} a_{\iota} z^{\iota-u-i}
$$

Since $q$-calculus is extensively used in various fields of mathematics and physics, researchers are very interested in it. The versatile applications of the $q$-derivative operator $\mathrm{T}_{q}$ make it exceptionally important in the study of Geometric Function Theory. Ismail et al.[18,19] proposed the concept of a $q$-extension of the class of starlike functions for the first time in 1990. In [20], Srivastava, on the other hand, used the $q$-calculus principles in his work, which was published by systematically employing simple (or $q$ ) hypergeometric functions:

$$
\tau \Upsilon_{\gamma}\left(\tau, \gamma \in \mathbb{N}_{0}=\{0,1,2, \ldots\}\right)
$$

For more details about the Theory of Geometric Functions (GFT), we refer to [20]. In recent years,in [21], Srivastava studied the state of the art survey and applications of $q$-calculus, $q$ derivative operator, fractional $q$-calculus, and fractional $q$-derivative operators in Geometric Function Theory of Complex Analysis in a survey-cum-expository research paper, as well as the apparent triviality of the so-called $(u, q)$-calculus involving redundant $q$-derivative operators.

Important research has played a role in the development of the geometric function theory of complex analysis, it is influenced by the previously mentioned works. Many researchers surveyed in the aforementioned work by Srivastava [22]. They have identified several convolutionary and fractional calculus $q$-operators. Some of the recent developments are briefly mentioned for the first time. Mahmood et al. in [23], and authors in [24,25] have obtained a number of interesting results for $q$-star functions related to the conical field. One can refer to [26,27], and the references therein for relevant results. Furthermore, Srivastava's recently published a review article which is potentially useful for researchers and academics that are interested in these subjects [21]. One may refer to [11,27] for other recent research involves the calculus of $q$. Our aim in this article is primarily to generalize the work that is discussed in [28]. 
Definition (1.3): For functions $g \in \Sigma(u)$ in the class $\mathcal{M} S_{q}^{*}[u, b, i ; C, D]$ if and only if

$$
\left|\frac{\frac{w^{b}\left(\mathrm{~T}_{q}^{(b+i)} g\right)(w)}{\left(\mathrm{T}_{q}^{(i)} g\right)(w)}+1}{w^{b}(D+1) \frac{\left(\mathrm{T}_{q}^{(b+i)} g\right)(w)}{\left(\mathrm{T}_{q}^{(i)} g\right)(w)}+(C+1)}\right|<1-\beta,
$$

If there are sufficiently small coefficients. We will assume throughout this document, unless otherwise stated,

$-1 \leq D<C \leq 1$ and $q \in(0,1)$.

\section{Main Results}

In this section, we provide an appropriate condition for a $g$ function to be in the $\mathcal{M} S_{q}^{*}[u, b, i ; C, D]$ class, which will work as one of the key results to find other results of this paper.

Theorem (2.1): The $g(w) \in M \Sigma(u)$ function of the form (1.1)is in the $\operatorname{class} \mathcal{M} S_{q}^{*}[u, b, i ; C, D]$ if it fulfills the following condition:

$$
\begin{aligned}
& \sum_{l=1}^{\infty}\left[\left(\zeta_{(3, \iota)}+\zeta_{(4, \iota)}\right)+\right.\left.(1-\beta)\left[|D+1| \zeta_{(3, \iota)}+|C+1| \zeta_{(4, \imath)}\right]\right]\left|a_{\iota}\right| \\
& \leq(\beta-1)\left[|D+1| \zeta_{(1)}+|C+1| \zeta_{(2)}\right]-\left(\zeta_{(1)}+\zeta_{(2)}\right)
\end{aligned}
$$

where

$$
\zeta_{(1)}=\frac{[-u]_{q} !}{[-u-i-b]_{q} !}, \quad \zeta_{(2)}=\frac{[-u]_{q} !}{[-u-i]_{q} !},
$$

and

$$
\zeta_{(3, \iota)}=\frac{[\iota-u]_{q} !}{[\iota-u-i-b]_{q} !}, \quad \zeta_{(4, \iota)}=\frac{[\iota-u]_{q} !}{[\iota-u-i]_{q} !}
$$

Proof. If the inequality (2.9) is true, it is sufficient to demonstrate that

$$
\begin{aligned}
& \left|\frac{\frac{w^{b}\left(\mathrm{~T}_{q}^{(b+i)} g\right)(w)}{\left(\mathrm{T}_{q}^{(i)} g\right)(w)}+1}{w^{b}(D+1) \frac{\left(\mathrm{T}_{q}^{(b+i)} g\right)(w)}{\left(\mathrm{T}_{q}^{(i)} g\right)(w)}+(C+1)}\right|<1-\beta, \\
& =\left|\frac{w^{b}\left(\mathrm{~T}_{q}^{(b+i)} g\right)(w)+\left(\mathrm{T}_{q}^{(i)} g\right)(w)}{w^{b}(D+1)\left(\mathrm{T}_{q}^{(b+i)} g\right)(w)+(C+1)\left(\mathrm{T}_{q}^{(i)} g\right)(w)}\right| \\
& \left.=\mid \frac{w^{b}\left[\frac{[-u]_{q} !}{[-u-i-b]_{q} !} w^{-u-i-b}+\sum_{l=1}^{\infty} \frac{[\iota-u]_{q} !}{[\iota-u-i-b]_{q} !} a_{\iota} z^{\iota-u-i-b}\right]+\frac{[-u]_{q} !}{[-u-i]_{q} !} w^{-u-i}+\sum_{\iota=1}^{\infty} \frac{[\iota-u]_{q} !}{[\iota-u-i]_{q} !} a_{\iota} z^{z^{-u-i}}}{w^{b}(D+1)\left[\frac{[-u]_{q} !}{[-u-i-b]_{q} !} w^{-u-i-b}+\sum_{\iota=1}^{\infty} \frac{[\iota-u]_{q} !}{[\iota-u-i-b]_{q} !} a_{\iota} z^{\iota-u-i-b}\right]+(C+1)\left[\frac{[-u]_{q} !}{[-u-i]_{q} !} w^{-u-i}+\sum_{\iota=1}^{\infty} \frac{[\iota-u]_{q} !}{[\iota-u-i]_{q} !} a_{\iota} z^{l-u-i}\right.}\right] \mid \\
& \leq \frac{\left(\zeta_{(1)}+\zeta_{(2)}\right)+\sum_{\iota=1}^{\infty}\left(\zeta_{(3, l)}+\zeta_{(4, \imath)}\right)\left|a_{\iota}\right|}{|-D-1| \zeta_{(1)}+|-C-1| \zeta_{(2)}+\sum_{\iota=1}^{\infty}\left[|-D-1| \zeta_{(3, l)}+|-C-1| \zeta_{(4, l)}\right]\left|a_{\iota}\right|},
\end{aligned}
$$

where $\zeta_{(1)}, \zeta_{(2)}, \zeta_{(3, l)}$ and $\zeta_{(4, l)}$ are given by (2.10) and (2.11), respectively. We see that if the condition in (2.9) is fulfilled, then $1-\beta$ is the upper limit of the last expression in (2.12). This completes the evidence of Theorem (2.1).

Corollary (2.2): If the class $\mathcal{M} S_{q}^{*}[u, b, i ; C, D]$ contains a function $g(w) \in M \Sigma(u)$ which is defined by (1.1), then 


$$
\left|a_{\iota}\right| \leq \frac{(\beta-1)\left[|D+1| \zeta_{(1)}+|C+1| \zeta_{(2)}\right]-\left(\zeta_{(1)}+\zeta_{(2)}\right)}{\left[\left(\zeta_{(3, l)}+\zeta_{(4, l)}\right)+(1-\beta)\left[|D+1| \zeta_{(3, l)}+|C+1| \zeta_{(4, l)}\right]\right]},
$$

with equality for each $\iota$, the form's function

$$
g_{\iota}(w)=w^{-u}+\frac{(\beta-1)\left[|D+1| \zeta_{(1)}+|C+1| \zeta_{(2)}\right]-\left(\zeta_{(1)}+\zeta_{(2)}\right)}{\left[\left(\zeta_{(3, l)}+\zeta_{(4, l)}\right)+(1-\beta)\left[|D+1| \zeta_{(3, l)}+|C+1| \zeta_{(4, l)}\right)\right]} w^{\iota-u} .
$$

Now, for simplicity, the following distortion theorem for the function class $\mathcal{M} S_{q}^{*}[u, b, i ; C, D]$ is stated and shown.

Theorem (2.3): If $g(w) \in \mathcal{M} S_{q}^{*}[u, b, i ; C, D]$ then for $\left|w^{-u}\right|=r^{-u}$, we get

$$
|g(w)| \geq r^{-u}-\left[\frac{(\beta-1)\left[|D+1| \zeta_{(1)}+|C+1| \zeta_{(2)}\right]-\left(\zeta_{(1)}+\zeta_{(2)}\right)}{\left[\left(\zeta_{(3,1)}+\zeta_{(4,1)}\right)+(1-\beta)\left[|D+1| \zeta_{(3,1)}+|C+1| \zeta_{(4,1)}\right]\right]}\right] r^{-u+1},
$$

and

$$
|g(w)| \leq r^{-u}+\left[\frac{(\beta-1)\left[|D+1| \zeta_{(1)}+|C+1| \zeta_{(2)}\right]-\left(\zeta_{(1)}+\zeta_{(2)}\right)}{\left[\left(\zeta_{(3,1)}+\zeta_{(4,1)}\right)+(1-\beta)\left[|D+1| \zeta_{(3,1)}+|C+1| \zeta_{(4,1)}\right]\right]}\right] r^{-u+1} .
$$

Equalities in (2.14) and (2.15) for the $g(w)$ function are achieved given by

$$
g(w)=w^{-u}-\frac{(\beta-1)\left[|D+1| \zeta_{(1)}+|C+1| \zeta_{(2)}\right]-\left(\zeta_{(1)}+\zeta_{(2)}\right)}{\left[\left(\zeta_{(3,1)}+\zeta_{(4,1)}\right)+(1-\beta)\left[|D+1| \zeta_{(3,1)}+|C+1| \zeta_{(4,1)}\right]\right]} w^{-u+1},
$$

at $\quad w=r$ and $w=r e^{(i(2 \ell+1) \pi)} \quad \ell \in\{0, \pm 1, \pm 2, \ldots\}$, where $\zeta_{(1)}, \zeta_{(2)}, \zeta_{(3, l)}$ and $\zeta_{(4, l)}$ are given by (2.10) and (2.11), respectively.

Proof. By Theorem (2.1).

$$
\begin{gathered}
{\left[\left(\zeta_{(3,1)}+\zeta_{(4,1)}\right)+(1-\beta)\left[|D+1| \zeta_{(3,1)}+|C+1| \zeta_{(4,1)}\right]\right] \sum_{l=1}^{\infty}\left|a_{\iota}\right|} \\
\leq\left[\left(\zeta_{(3, l)}+\zeta_{(4, l)}\right)+(1-\beta)\left[|D+1| \zeta_{(3, l)}+|C+1| \zeta_{(4, \imath)}\right]\right] \sum_{l=1}^{\infty}\left|a_{\iota}\right| \\
\leq(\beta-1)\left[|D+1| \zeta_{(1)}+|C+1| \zeta_{(2)}\right]-\left(\zeta_{(1)}+\zeta_{(2)}\right),
\end{gathered}
$$

which yields:

$$
\begin{aligned}
|g(w)| \geq r^{-u} & -\sum_{\iota=1}^{\infty}\left|a_{\iota}\right| r^{\imath-u} \geq r^{-u}-r^{-u+1} \sum_{l=1}^{\infty}\left|a_{\iota}\right| \\
& \geq r^{-u}-\left[\frac{(\beta-1)\left[|D+1| \zeta_{(1)}+|C+1| \zeta_{(2)}\right]-\left(\zeta_{(1)}+\zeta_{(2)}\right)}{\left[\left(\zeta_{(3,1)}+\zeta_{(4,1)}\right)+(1-\beta)\left[|D+1| \zeta_{(3,1)}+|C+1| \zeta_{(4,1)}\right]\right]}\right] r^{-u+1}
\end{aligned}
$$

and

$$
\begin{aligned}
|g(w)| \leq r^{-u} & +\sum_{\iota=1}^{\infty}\left|a_{\iota}\right| r^{\iota-u} \leq r^{-u}+r^{-u+1} \sum_{l=1}^{\infty}\left|a_{\iota}\right| \\
& \leq r^{-u}+\left[\frac{(\beta-1)\left[|D+1| \zeta_{(1)}+|C+1| \zeta_{(2)}\right]-\left(\zeta_{(1)}+\zeta_{(2)}\right)}{\left[\left(\zeta_{(3,1)}+\zeta_{(4,1)}\right)+(1-\beta)\left[|D+1| \zeta_{(3,1)}+|C+1| \zeta_{(4,1)}\right]\right]}\right] r^{-u+1} .
\end{aligned}
$$

Thus, we have completed the evidence of Theorem (2.3).

If $b=-1=i+1=u$ and make $q \rightarrow 1-$, then special case of Theorem (2.3) coincides with as follows. A comparable outcome is provided in [19]. 
Corollary (2.4):[19]. If $g(w) \in \mathcal{M} S^{*}[\beta]$, then

$$
r-\left(\frac{1-\beta}{2-\beta}\right) r^{2} \leq|g(w)| \leq r+\left(\frac{1-\beta}{2-\beta}\right) r^{2} . \quad(|w|=r, \quad(0<r<1))
$$

The evidence of the following outcome is equivalent to that of Theorem (2.3), so the analogous specifics of our Theorem (2.5) evidence have been omitted.

Theorem (2.5): If $g(w) \in \mathcal{M} S_{q}^{*}[u, b, i ; C, D]$ then for $\left|w^{-u}\right|=r^{-u}$, we get

$$
-\left[\frac{(-u+1)(\beta-1)\left[|D+1| \zeta_{(1)}+|C+1| \zeta_{(2)}\right]-\left(\zeta_{(1)}+\zeta_{(2)}\right)}{\left[\left(\zeta_{(3,1)}+\zeta_{(4,1)}\right)+(1-\beta)\left[|D+1| \zeta_{(3,1)}+|C+1| \zeta_{(4,1)}\right]\right]}\right] r^{-u},(2.17)
$$

and

$$
+\left[\frac{(-u+1)(\beta-1)\left[|D+1| \zeta_{(1)}+|C+1| \zeta_{(2)}\right]-\left(\zeta_{(1)}+\zeta_{(2)}\right)}{\left[\left(\zeta_{(3,1)}+\zeta_{(4,1)}\right)+(1-\beta)\left[|D+1| \zeta_{(3,1)}+|C+1| \zeta_{(4,1)}\right]\right]}\right] r^{-u} \cdot(2.18)
$$

For the function $g(w)$ given by $(2.16)$, the outcome is sharp.

When we bring it in, $b=-1=i+1=u$ and let $q \rightarrow 1-$, in its special case, Theorem (2.3) reduces to the following known result.

Corollary (2.6):[19]. If $g(w) \in \mathcal{M} S^{*}[\beta]$, then

$$
1-\left(\frac{2(1-\beta)}{2-\beta}\right) r \leq\left|g^{\prime}(w)\right| \leq 1+\left(\frac{2(1-\beta)}{2-\beta}\right) r . \quad(|w|=r \quad(0<r<1)) .
$$

Finally, for functions belong to the family $\mathcal{M} S_{q}^{*}[u, b, i ; C, D]$, we measure the radius of closeto-convexity, starlikeness and convexity.

Theorem (2.7): If the class $\mathcal{M} S_{q}^{*}[u, b, i ; C, D]$ contains a function $g(w)$ that is defined by (1.2). Then $g(w)$ is a meromorphic u-valent close-to-convex function of order $\Lambda(0 \leq \Lambda<$ $u)$ for $|w| \leq r_{0}(u, \iota, \alpha, \Lambda)$, where

$$
r_{0}=\inf _{\iota \geq 1}\left[\frac{\left[\left(\zeta_{(3,1)}+\zeta_{(4,1)}\right)+(1-\beta)\left[|D+1| \zeta_{(3,1)}+|C+1| \zeta_{(4,1)}\right]\right](-u-\Lambda)}{(\beta-1)\left[|D+1| \zeta_{(1)}+|C+1| \zeta_{(2)}\right]-\left(\zeta_{(1)}+\zeta_{(2)}\right)(\iota-u)}\right]^{\frac{1}{\iota}} \text {. }
$$

For the function $g_{l}(w)$ given by (13), the outcome is sharp.

Proof. Via the implementation of Theorem (2.1) and the form(1.2), we see for $|w|<r_{0}$ that

$$
\left|\frac{g^{\prime}(w)}{w^{-u-1}}+u\right|<u-\Lambda . \quad\left(|w| \leq r_{0}\right)
$$

It completes the evidence of Theorem (2.7).

Theorem (2.8): If the class $\mathcal{M} S_{q}^{*}[u, b, i ; C, D]$ contains a function $g(w)$ that is defined by (1.2). Then $g(w)$ is a meromorphic u-valent starlike function of order $\Lambda(0 \leq \Lambda<u)$ for $|w| \leq r_{1}(u, \iota, \alpha, \Lambda)$, where

$$
r_{1}=\inf _{\iota \geq 1}\left[\frac{\left[\left(\zeta_{(3,1)}+\zeta_{(4,1)}\right)+(1-\beta)\left[|D+1| \zeta_{(3,1)}+|C+1| \zeta_{(4,1)}\right]\right](-u-\Lambda)}{(\beta-1)\left[|D+1| \zeta_{(1)}+|C+1| \zeta_{(2)}\right]-\left(\zeta_{(1)}+\zeta_{(2)}\right)(\iota-u-\Lambda)}\right]^{\frac{1}{\iota}} .
$$

For the function $g_{\imath}(w)$ is given by (2.13), the outcome is sharp.

Proof. It is shown, using the same measures as in the Theorem Proof (2.7), that

It completes the evidence of Theorem (2.8).

$$
\left|\frac{w g^{\prime}(w)}{g(w)}+u\right|<u-\Lambda . \quad\left(|w| \leq r_{1}\right)
$$


Corollary (2.9): If the class $\mathcal{M} S_{q}^{*}[u, b, i ; C, D]$ contains a function $g(w)$ that is defined by (1.2). Then $g(w)$ is a meromorphic u-valent starlike function of order $\Lambda(0 \leq \Lambda<u)$ for $|w| \leq r_{2}(u, \iota, \alpha, \Lambda)$, where

$$
=\inf _{\iota \geq 1}\left[\frac{\left[\left(\zeta_{(3,1)}+\zeta_{(4,1)}\right)+(1-\beta)\left[|D+1| \zeta_{(3,1)}+|C+1| \zeta_{(4,1)}\right]\right]-u(-u-\Lambda)}{(\beta-1)\left[|D+1| \zeta_{(1)}+|C+1| \zeta_{(2)}\right]-\left(\zeta_{(1)}+\zeta_{(2)}\right)(\iota-u)(\iota-u-\Lambda)}\right]^{\frac{1}{\iota}} .
$$

For the function $\mathcal{g}_{j}(w)$ is given by (2.13), the outcome is sharp.

\section{Conclusions}

In the punctured unit disk $\mathcal{L}^{*}$, we have been systematically introduced and analyzed some important subclasses of meromorphically multivalent $q$-starlike functions. We have also given important links to the different findings, as well as in this paper we have also shown some important results in this field of study.

\section{References}

[1] Yang, D. G. and Liu, J. L. "Some sufficient conditions for p-valent strongly starlike functions", Comput. Math., Appl., 59, 2018-2025, 2010.

[2] Hussein, A. A. and Jassim, K. A. H. "On A Certain Class of Meromorphic Multivalent Functions Defined by Fractional Calculus Operator”. Iraqi Journal of Science, 2685-2696, 2019.

[3] Silverman, H. "Univalent functions with negative coefficients", Proc. Am. Math. Soc., 51, 109116, 1975.

[4] Srivastava, H. M., Ahmad, Q. Z., Khan, N., Kiran, S. and Khan, B. "Some applications of higherorder derivatives involving certain subclass of analytic and multivalent functions", J. Nonlinear Var., Anal., 2, 343-353, 2018.

[5] Altintas, O. "Neighborhoods of certain p-valently analytic functions with negative coefficients", Appl. Math. Comput., 187, 47-53, 2007.

[6] Jassim, K. A. H. and Hussein, S. K. "On a Class of Meromorphic Multiivalent Functiions Convoluted withi Higher Derivatiives of Fractionali Calculus Operator", Iraqi Journal of Science, 2237-2245, 2019.

[7] Hameed, M. I., Shihab, B. N. and Jassim, K. A. "Certain Geometric Properties of Meromorphic Functions Defined by a New Linear Differential Operator", In Journal of Physics: Conference Series, Vol. 1999, No. 1, p. 012090, IOP Publishing, 2021.

[8] Atshan, W. G., Battor, A. H. and Abaas, A. F. "Some sandwich theorems for meromorphic univalent functions defined by new integral operator", Journal of Interdisciplinary Mathematics, 24(3), 579-591, 2021.

[9] AL-Ameedee, S. A. Atshan, W. G. and AL-Maamori, F. A. "On sandwich results of univalent functions defined by a linear operator", Journal of Interdisciplinary Mathematics, 23(4), 803-809, 2020.

[10] Juma, A. R. S., Hameed, R. A. and Hameed, M. I. "Some Results of Second Order Differential Subordination Involving Generalized Linear Operator", Acta Universitatis Apulensis, 53, 19-39, 2018.

[11] Khan, Q., Arif, M., Raza, M., Srivastava, G. and H. Tang, H. "Some applications of a new integral operator in q-analog for multivalent functions", Mathematics, 7, 1178, 2019.

[12] Khan, B., Liu, Z.G., Srivastava, H.M.,. Khan, N., Darus, M. and Tahir, M. "A study of some families of multivalent q-starlike functions involving higher-order q-derivatives", Mathematics,8(9), 1470, 2020.

[13] Liu, J. L. and Srivastava, H. M. "Some convolution conditions for starlikeness and convexity of meromorphically multivalent functions", Appl. Math. Lett., 16, 13-16, 2003.

[14] Gasper, G. and Rahman, M. "Basic Hypergeometric Series", Cambridge University Press, Cambridge, 1990. 
[15] Hameed, M. and Ibrahim, I. "Some Applications on Subclasses of Analytic Functions Involving Linear Operator", 2019 International Conference on Computing and Information Science and Technology and Their Applications (ICCISTA), IEEE, 2019.

[16] Jackson, F. H. "On q-definite integrals”, Q. J. Pure Appl. Math. 41, 193-203, 1910.

[17] Jackson, F. H. “ $q$-Difference equations”, Am. J. Math. 32, 305-314, 1910.

[18] Hameed, M. I., Ozel, C., Al-Fayadh, A. and Juma, A. R. S. "Study of certain subclasses of analytic functions involving convolution operator", AIP Conference Proceedings, Vol. 2096. No. 1. AIP Publishing LLC, 2019.

[19] Silverman, H. "Univalent functions with negative coefficients", Proc. Am. Math. Soc., 51, 109116, 1975.

[20] Srivastava, H. M. "Univalent functions, fractional calculus and associated generalized hypergeometric functions", Chichester, UK, JohnWiley and Sons, New York, NY, USA, 329354, 1989.

[21] Srivastava, H. M. "Operators of basic (or q-) calculus and fractional q-calculus and their applications in geometric function theory of complex analysis", Iran. J. Sci. Technol. Trans. A Sci., 44, 327 344, 2020.

[22] Srivastava, H. M., Tahir, M., Khan, B., Ahmad, Q. Z. and Khan, N. "Some general families of qstarlike functions associated with the Janowski functions", Filomat, 33, 2613-2626, 2019.

[23] Mahmood, S., Srivastava, H. M., Khan, N., Ahmad, Q. Z., Khan, B. and Ali, I. "Upper bound of the third Hankel determinant for a subclass of q-starlike functions", Symmetry, 11, 347, 2019.

[24] Hameed, M. I. and Ali, M. H. "Some Classes Of Analytic Functions For The Third Hankel Determinant", In Journal of Physics: Conference Series, Vol. 1963, No. 1, p. 012080, IOP Publishing, 2021.

[25] Srivastava, H. M., Khan, B., Khan, N., Ahmad, Q. Z. and Tahir, M. "A generalized conic domain and its applications to certain subclasses of analytic functions", Rocky Mountain J. Math., 49, 2325-2346, 2019.

[26] Mahmood, S., Ahmad, Q .Z., Srivastava, H. M., Khan, N., Khan, B. and Tahir, M. "A certain subclass of meromorphically q-starlike functions associated with the Janowski functions", J. Inequal. Appl., 88, (1), 1-11, 2019.

[27] Shi, L., Khan, Q., Srivastava, G., Liu, J.L. and Arif, M. "A study of multivalent q-starlike functions connected with circular domain", Mathematics, 7, 1-12, 2019.

[28] Wongsaijai, B. and Sukantamala, N. "Certain properties of some families of generalized starlike functions with respect to q-calculus”, Abstr. Appl. Anal., 6180140, 2016. 